\title{
Heart rate variability during simulated hemorrhage with lower body negative pressure in high and low tolerant subjects
}

\author{
Carmen Hinojosa-Laborde ${ }^{1}{ }^{*}$, Caroline A. Rickards ${ }^{2}$, Kathy L. Ryan ${ }^{1}$ and Victor A. Convertino ${ }^{1}$ \\ 1 US Army Institute of Surgical Research, Fort Sam Houston, TX, USA \\ ${ }^{2}$ Department of Health and Kinesiology, University of Texas at San Antonio, San Antonio, TX, USA
}

Edited by:

Heikki Veli Huikuri, University of Oulu, Finland

\section{Reviewed by:}

Mikko Paavo Tulppo, Verve, Finland

Phyllis Kravet Stein, Washington

University School of Medicine, USA

${ }^{*}$ Correspondence:

Carmen Hinojosa-Laborde, U.S. Army

Institute of Surgical Research, 3698

Chambers Pass, Fort Sam Houston,

TX 78234-6315, USA.

e-mail: c.hinojosalaborde@us.army.mil
Heart rate variability (HRV) decreases during hemorrhage, and has been proposed as a new vital sign to assess cardiovascular stability in trauma patients. The purpose of this study was to determine if any of the HRV metrics could accurately distinguish between individuals with different tolerance to simulated hemorrhage. Specifically, we hypothesized that (1) HRV would be similar in low tolerant (LT) and high tolerant (HT) subjects at presyncope when both groups are on the verge of hemodynamic collapse; and (2) HRV could distinguish LT subjects at presyncope from hemodynamically stable HT subjects (i.e., at a submaximal level of hypovolemia). Lower body negative pressure (LBNP) was used as a model of hemorrhage in healthy human subjects, eliciting central hypovolemia to the point of presyncopal symptoms (onset of hemodynamic collapse). Subjects were classified as LT if presyncopal symptoms occurred during the -15 to $-60 \mathrm{mmHg}$ levels of LBNP, and HT if symptoms occurred after LBNP of $-60 \mathrm{mmHg}$. A total of $20 \mathrm{HRV}$ metrics were derived from $\mathrm{R}-\mathrm{R}$ interval measurements at the time of presyncope, and at one level prior to presyncope (submax) in LT and HT groups. Only four HRV metrics (Long-range Detrended Fluctuation Analysis, Forbidden Words, Poincaré Plot Descriptor Ratio, and Fractal Dimensions by Curve Length) supported both hypotheses. These four HRV metrics were evaluated further for their ability to identify individual LT subjects at presyncope when compared to HT subjects at submax. Variability in individual LT and HT responses was so high that LT responses overlapped with $\mathrm{HT}$ responses by $85-97 \%$. The sensitivity of these HRV metrics to distinguish between individual LT from HT subjects was 6-33\%, and positive predictive values were $40-73 \%$. These results indicate that while a small number of HRV metrics can accurately distinguish between LT and HT subjects using group mean data, individual HRV values are poor indicators of tolerance to hypovolemia.

Keywords: lower body negative pressure, hypovolemia, hemorrhage, heart rate variability, heart period variability

\section{INTRODUCTION}

In trauma patients, the primary cause of death within the first hour of injury is hemorrhage (Bellamy, 1984; Sauaia et al., 1995; Champion et al., 2003). Early detection of hypovolemia in trauma patients is therefore critical to improving triage and providing life saving interventions (LSIs). Unfortunately, the severity of hemorrhage or central hypovolemia is difficult to detect by first responders or other emergency medical personnel, as the traditional vital signs currently available to them, such as arterial pressure, heart rate, respiration rate, and pulse character do not consistently change until hemorrhage has progressed to the point of decompensation (i.e., hemodynamic collapse; Heckbert et al., 1998; Parks et al., 2006; Convertino et al., 2008). Assessing the severity of hemorrhage in trauma patients is further complicated by the reality that there are individuals who have a low tolerance to hemorrhage such that, for the same level of blood loss, low tolerant (LT) individuals reach the point of cardiovascular collapse in less time than those who are high tolerant (HT; Shoemaker et al., 1973, 2006; Klemcke et al., 2011). Thus, there is an urgent need to identify physiological parameters that can accurately track hypovolemia in trauma patients, and have the sensitivity to provide early identification of a patient that has a low tolerance to this physiological insult. New vital signs are needed which can detect the onset, progression, and severity of hypovolemia in individual patients.

The measurement of the variation in R-R intervals, i.e., heart rate variability (HRV; Malik, 1996), can provide information regarding overall cardiovascular status. For instance, healthy individuals with normal autonomic function have high HRV, while individuals with a stressed cardiovascular system (via activity, disease, or dysfunction) can be identified by reduced HRV (Thayer and Sternberg, 2006). HRV has been extensively studied in hospital settings such as intensive care units to assess cardiovascular status and predict mortality in trauma patients (Winchell and Hoyt, 1996, 1997; Grogan et al., 2005; Norris et al., 2005, 2008; Morris et al., 2006). Considering that monitoring electrocardiogram 
(ECG) signals is non-invasive, can be accomplished in all echelons of care (including the pre-hospital environment), and can potentially provide information regarding cardiovascular status, the utility of HRV as a pre-hospital triage assist tool is the focus of many investigations (Cooke et al., 2006a,b; Batchinsky et al., 2007a; Cancio et al., 2008; Ryan et al., 2011).

One criterion for the development of accurate and timely assessment of the clinical status of patients with hemorrhage is the capability to investigate the continuous physiological response to progressive reductions in central blood volume. This capability has become available with the emergence of lower body negative pressure (LBNP) as a human experimental model for hemorrhage (Cooke et al., 2004). Cardiovascular responses during LBNP are reproducible, and are consistent with those observed during hemorrhage (Convertino and Sather, 2000; Convertino, 2001; Cooke et al., 2004). In addition, LBNP allows for the assessment of cardiovascular responses which are specific to hypovolemia as confounding factors associated with trauma such as pain and inflammation are absent. Thus, the controlled experimental environment of LBNP allows for optimal conditions in which to study HRV. Using the LBNP model to induce a clinical endpoint (i.e., presyncope), human subjects can be identified as having HT or LT to central hypovolemia (Sather et al., 1986; Convertino and Sather, 2000; Rickards et al., 2011). If HRV metrics reflect the status of cardiovascular stability during progressive central hypovolemia, then LT subjects would be expected to display greater reductions in HRV compared to HT subjects. We therefore used LBNP tolerance as a model to test the hypotheses that: (1) HRV at presyncope is similar in LT and HT subjects when both groups are on the verge of cardiovascular collapse, and (2) LT subjects have lower HRV at presyncope compared to HT subjects who remain hemodynamically stable.

\section{MATERIALS AND METHODS SUBJECTS}

This study was conducted under a protocol reviewed and approved by the Brooke Army Medical Center and US Army Medical Research and Materiel Command Institutional Review Boards and in accordance with the approved protocol. All studies were conducted at the U.S. Army Institute of Surgical Research, Fort Sam Houston, TX, USA. Normotensive, non-smoking healthy human volunteers $(N=120$; male $=75$, female $=45$, age $28 \pm 1$ years; height $174 \pm 1 \mathrm{~cm}$; weight $76 \pm 1 \mathrm{~kg}$ ) participated in this study after evaluation of their medical history and physical examination by a physician to ensure the absence of previous and current medical conditions that would exempt them as participants. All female subjects were confirmed as not pregnant by a urine pregnancy test conducted within an hour before the experiment. For $24 \mathrm{~h}$ prior to the study, participants were instructed to maintain their normal sleep patterns, refrain from exercise, and abstain from alcohol and autonomic stimulants including prescription and non-prescription drugs such as caffeine, alcohol, and decongestants. The potential effect of caffeine withdrawal on baseline hemodynamics was not assessed in this study. Subjects were given a written description of the experimental protocol and the risks associated with the study. On the day of the study, the subjects were made familiar with the laboratory, given a verbal briefing on the protocol and procedures, and encouraged to ask questions of the investigators. All subjects signed an IRB approved informed consent form.

\section{LBNP PROTOCOL}

Subjects were instrumented for standard lead II ECG to record $\mathrm{R}-\mathrm{R}$ intervals (RRI), and a finger cuff to record beat-by-beat finger arterial pressure by photoplethysmography (Finometer Blood Pressure Monitor, TNO-TPD Biomedical Instrumentation, Amsterdam, The Netherlands). To simulate hemorrhage in conscious humans, central hypovolemia was induced by application of LBNP. Previous studies have shown that the LBNP protocol can closely simulate the hemodynamic challenges associated with pre-shock hemorrhage (Cooke et al., 2004; Ward et al., 2010). Subjects were positioned supine within an airtight chamber that was sealed at the level of the iliac crest by a neoprene skirt. The LBNP protocol consisted of a 5 -min control period (baseline) followed by $5 \mathrm{~min}$ of chamber decompression at $-15,-30,-45$, and $-60 \mathrm{mmHg}$, and then additional increments of $-10 \mathrm{mmHg}$ every $5 \mathrm{~min}$ until the onset of cardiovascular collapse followed by a 10-min recovery period. The application of LBNP was terminated based on three criteria.: (a) sudden onset of relative bradycardia, (b) progressive fall of systolic pressure below $80 \mathrm{mmHg}$; and, (c) voluntary subject termination due to the onset of presyncopal symptoms such as sweating, nausea, dizziness, vision alterations, or general discomfort. During the LBNP protocol, arterial pressure waveform (Finometer) and ECG signals were sampled continuously at $500 \mathrm{~Hz}$ and digitally recorded with WinDaq data acquisition software (DATAQ Instruments, Akron, OH, USA).

\section{HIGH TOLERANCE VS. LOW TOLERANCE}

Subjects were categorized as LT or HT based on the level of LBNP at which they experienced the onset of cardiovascular collapse (Sather et al., 1986; Rickards et al., 2011). LT subjects experienced cardiovascular collapse before or during $-60 \mathrm{mmHg}$ of LBNP, and HT subjects experienced cardiovascular collapse after $-60 \mathrm{mmHg}$ of LBNP.

\section{DATA ANALYSIS}

Arterial pressure waveform and ECG signals were analyzed during the last 3 min of each LBNP level using a commercially available software program (WinCPRS, Absolute Aliens, Turku, Finland). Beat-to-beat stroke volume was derived from the arterial pressure waveform using the pulse contour method (Jansen et al., 1990).

Electrocardiogram signals were visually inspected and confirmed to be free of noise, persistent ectopic beats, or arrhythmias. When ectopic beats were identified, the presence of a single event within the 3-min analysis window resulted in the correction of the beat by linear interpolation prior to analysis. If more than one ectopic beat occurred during an LBNP level, the ECG data for that level were not analyzed. Acceptable ECG signals were used to calculate RRI and heart rate. The RRI signal was used to derive 20 indices of HRV described in detail in previous publications from this laboratory (Rickards et al., 2010a; Ryan et al., 2010). Linear methods were used to calculate HRV metrics in both the time domain [ $n=9$; RRI standard deviation (RRISD), RRI root mean squared standard deviation (RMSSD), percentage adjacent RRIs varying by at least $50 \mathrm{~ms}$ (pNN50), Poincaré 
plot descriptors standard deviation 1 and standard deviation 2 (SD1 and SD2), SD1/SD2 ratio, SD2/SD1 ratio, complex demodulation high frequency (CDM HF), complex demodulation-low frequency (CDM LF)]; and frequency domain $[n=2$; RRI low frequency power (RRI LF), RRI high frequency power (RRI HF)]. For frequency domain analysis, the RRI signals were plotted using linear interpolation, sampled at $5 \mathrm{~Hz}$, and then passed through a low-pass impulse response filter with a cutoff frequency of $0.5 \mathrm{~Hz}$. Data sets were submitted to a Fourier transform with a Hanning window. Non-linear methods were used to calculate heart rate complexity metrics from the RRI signal ( $n=9$; sample entropy (SampEn), Lempel-Ziv entropy (LZEn), fractal dimensions by curve length (FD-L), fractal dimensions by dispersion analysis (FD-DA), symbol dynamics entropy (SymDyn), normalized symbol dynamics entropy (DisnEn), long-range detrended fluctuation analysis (DFA long), short-range detrended fluctuation analysis (DFA short), and Forbidden Words (FW).

Heart rate variability metrics were compared in LT and HT subjects at the level of LBNP at which cardiovascular collapse was imminent (presyncope). In HT subjects, HRV metrics were also calculated one level of LBNP prior to the presyncopal level (submax), and compared with LT presyncopal values. HRV metrics which measured equal variability in LT and HT at presyncope and also less variability in LT at presyncope compared to HT at submax were further evaluated for their ability to distinguish between individual LT and HT subjects.

\section{SENSITIVITY AND POSITIVE PREDICTIVE VALUE}

The effectiveness of HRV metrics to accurately determine group membership (HT or LT) was evaluated by calculating sensitivity and positive predictive value. Sensitivity assesses the ability of a HRV metric to correctly identify a LT subject from all the subjects that were actually LT (i.e., true positives/true positives + false negatives). Positive predictive value is a measure of the ability of a HRV metric to identify a subject as LT from all the subjects that "appear" to be LT (i.e., true positives/true positives + false positives). A logistic regression model was used to evaluate the validity of HRV metrics as predictors of low tolerance to hemorrhage. Using this model, cutoff values were determined for each parameter at each level of LBNP based on receiver-operator characteristic analysis with specificity no less than 0.95 . Sensitivity and positive predictive values were then calculated based on this criterion (Pregibon, 1981).

\section{STATISTICAL ANALYSIS}

Data are expressed as mean \pm SE. Statistical analysis was conducted with commercially available software (SigmaStat; Systat Software, Richmond, CA, USA). Unpaired $t$-test, Chi-square, and $z$-test analyses were used to compare subject demographics and conditions at LBNP termination between tolerance groups. For hemodynamic responses, a two way analysis of variance with repeated measures on one variable was used to determine significant variable (tolerance and LBNP level) main effects $(p \leq 0.05)$. Subsequent multiple comparison tests (Holm-Sidak) determined significant differences between HT and LT groups $(p \leq 0.05)$. HRV measurements at presyncope and submax in LT and HT subjects were compared by unpaired $t$-test.

\section{RESULTS}

\section{SUBJECTS}

The LBNP procedure was conducted on 120 subjects. LBNP was terminated in 2 subjects during $-30 \mathrm{mmHg}$, in 5 subjects during $-45 \mathrm{mmHg}$, in 26 subjects during $-60 \mathrm{mmHg}$, in 43 subjects during $-70 \mathrm{mmHg}$, in 29 subjects during $-80 \mathrm{mmHg}$, in 13 subjects during $-90 \mathrm{mmHg}$, and in 2 subjects during $-100 \mathrm{mmHg}$. As a result, 33 subjects were identified as LT $(27.5 \%)$ and 87 subjects were identified as HT (72.5\%). The demographics and baseline mean arterial pressure and heart rates of these two groups of subjects were not statistically different (Table 1). However, baseline RRI was significantly lower in LT subjects compared to HT subjects. The presyncopal conditions observed at LBNP termination are shown in Table 2. Cardiovascular conditions include systolic pressure below $80 \mathrm{mmHg}$ and/or sudden onset of relative bradycardia, and presyncopal symptoms represent discomfort reported by the subject. LT and HT subjects experienced similar presyncopal conditions at LBNP termination.

\section{RRI TRACKS HYPOVOLEMIA}

Stroke volume and RRI at baseline $(\mathrm{LBNP}=0 \mathrm{mmHg})$ and during LBNP in LT and HT subjects are shown in Figures 1A,B. LT subjects experienced presyncopal conditions and/or symptoms before or during LBNP level of $-60 \mathrm{mmHg}$. HT subjects experienced presyncopal conditions and/or symptoms after $-60 \mathrm{mmHg}$.

Table 1 | Demographics and baseline hemodynamic variables for low tolerant and high tolerant subjects.

\begin{tabular}{llll}
\hline & Low tolerant & High tolerant & $P$ value \\
\hline$N$ & 33 & 87 & $<0.001$ \\
Male/female & $18 / 15$ & $57 / 30$ & 0.37 \\
Age (years) & $27 \pm 1$ & $29 \pm 1$ & 0.32 \\
Height (cm) & $173 \pm 2$ & $174 \pm 1$ & 0.60 \\
Weight (kg) & $76 \pm 3$ & $76 \pm 1$ & 0.98 \\
Mean arterial pressure (mmHg) & $99 \pm 2$ & $97 \pm 1$ & 0.23 \\
Heart rate (beats/min) & $69 \pm 2$ & $64 \pm 1$ & 0.06 \\
R-R interval (ms) & $896 \pm 22$ & $973 \pm 17$ & 0.01 \\
\hline
\end{tabular}

Data are expressed as mean $\pm S E$.

Table 2 | Prevalence of presyncopal conditions at LBNP termination in low tolerant and high tolerant subjects.

\begin{tabular}{llll}
\hline $\begin{array}{l}\text { Conditions at LBNP } \\
\text { termination }\end{array}$ & $\begin{array}{l}\text { Low tolerant } \\
(\boldsymbol{n}=\mathbf{3 3})\end{array}$ & $\begin{array}{l}\text { High tolerant } \\
(\boldsymbol{n}=\mathbf{8 7})\end{array}$ & $\boldsymbol{P}$ value \\
\hline Cardiovascular + symptoms & $17(52 \%)$ & $51(59 \%)$ & 0.622 \\
Cardiovascular only & $5(15 \%)$ & $12(14 \%)$ & 0.917 \\
Symptoms only & $11(33 \%)$ & $24(27 \%)$ & 0.694 \\
\hline
\end{tabular}

Cardiovascular conditions include systolic blood pressure below $80 \mathrm{mmHg}$ and/or sudden bradycardia. Symptoms represent presyncopal symptoms such as sweating, nausea, dizziness, vision alterations, or general discomfort as reported by the subject. Data are expressed as number of occurrences; group percentages are shown in parentheses. 


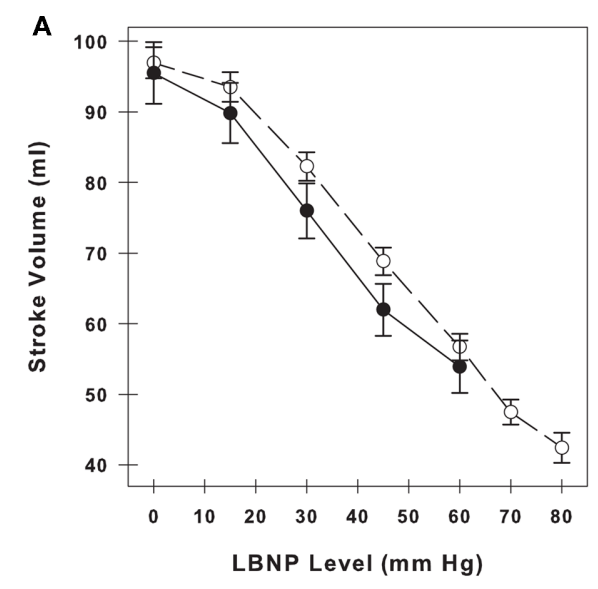

FIGURE 1 | Stroke volume (A) and R-R interval (B) during progressive lower body negative pressure (LBNP) in low tolerant subjects (closed circles, solid line) and high tolerant subjects (open circles, dashed line). At baseline, there are 33 low tolerant subjects and 87 high tolerant subjects.

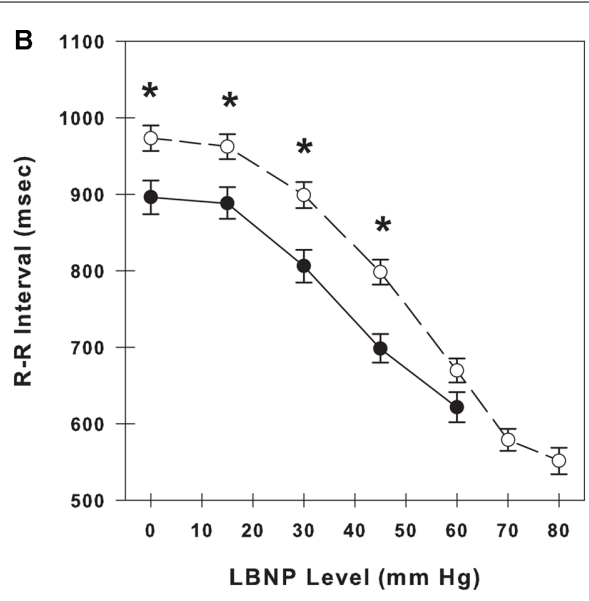

The number of subjects at the subsequent time points during LBNP decline as subjects experience presyncopal symptoms at various levels of LBNP. Data are shown as mean \pm SE. ${ }^{*}$ Denotes group differences at the same LBNP level $(p \leq 0.05)$.
Data for HT subjects are shown to LBNP level of $-80 \mathrm{mmHg}$. Both stroke volume and RRI decreased in a similar incremental fashion during the progression of LBNP, and average RRI measurements reflect and are correlated with the hypovolemia induced by LBNP (amalgamated $r^{2}=0.988$ between stroke volume and RRI). Stroke volume was not different between LT and HT groups, but RRI was significantly lower in LT subjects than HT subjects at rest and during $-15,-30$, and $-45 \mathrm{mmHg}$ LBNP $(p \leq 0.01)$.

\section{HRV MEASUREMENTS AT PRESYNCOPE AND SUBMAX LEVELS OF LBNP}

Figure 2 demonstrates the variation in RRI in a single LT subject and a single HT. These sample tracings of RRI were obtained during LBNP level of $-60 \mathrm{mmHg}$, and are $3 \mathrm{~min}$ in duration. The variation in RRI is low in the LT subject who is on the verge of cardiovascular collapse (at presyncope). In contrast, the variability in RRI is high in the HT subject who is hemodynamically stable (at submax).

Heart rate variability was quantified at presyncope in all LT and HT subjects, and at submax in HT subjects by 20 HRV metrics. These results are shown in Table 3. The first two columns in Table 3 indicate the parameter or HRV metric and its proportionality to variability. All HRV metrics are quantitatively proportional (P) to HRV except SD2/SD1, DFA long, DFA short, and FW, which are inversely proportional (I) to HRV. The values of proportional metrics decrease as variability decreases, while the values of inversely proportional metrics increase as variability decreases. Columns 3, 4, and 5 in Table 3 display the data for three groups: LT at presyncope, HT at presyncope, and HT at submax. The comparisons of variability between LT at presyncope verses HT at presyncope, and LT at presyncope verses HT at submax are also shown in Table 3, in columns 6 and 7, respectively. The hypotheses of our study were (1) that at presyncope HRV would be equal in LT and HT subjects (column 6) because both groups are on the verge of cardiovascular collapse; and (2)

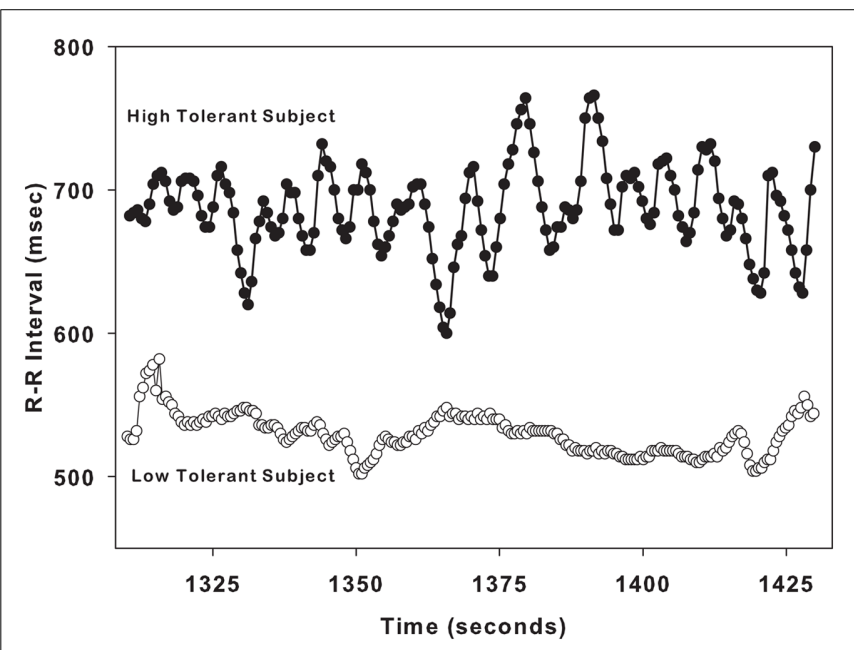

FIGURE 2 | Sample R-R interval tracings from one low tolerant subject (lower tracing, open circles) and one high tolerant subject (upper tracing, closed circles) are shown for a 2-min time span during LBNP level of $-60 \mathrm{mmHg}$.

that LT subjects would have less HRV at presyncope compared to HT subjects who remain hemodynamically stable at submax (column 7). These hypotheses are stated in Table 3 above the comparisons of HRV metrics (i.e., "LT $=$ HT," "LT < HT"). When comparing HRV between groups it is important to keep in mind that the numerical values of proportional metrics are consistent with variability (i.e., high value indicates high variability), but numerical values of metrics which are inversely proportional to variability are opposite to variability (i.e., high value indicates low variability). At presyncope, HRV was equivalent between LT and HT groups as measured by $7 \mathrm{HRV}$ metrics (bold type in column 6 of Table 3). When HRV was compared between LT subjects at presyncope and HT subjects at submax, 8 metrics were less 
Table 3 | HR, RRI, and HRV metrics in LT at presyncope, HT at presyncope, and HT at submax.

\begin{tabular}{|c|c|c|c|c|c|c|}
\hline Metric & $\begin{array}{l}\text { Proportional }(\mathrm{P}) \\
\text { Inversely proportional (I) }\end{array}$ & $\begin{array}{l}\text { LT at } \\
\text { presyncope }\end{array}$ & $\begin{array}{l}\text { HT at } \\
\text { presyncope }\end{array}$ & $\begin{array}{l}\text { HT at } \\
\text { submax }\end{array}$ & $\begin{array}{l}\text { LT @ Presyncope vs. } \\
\text { HT@ Presyncope }\end{array}$ & $\begin{array}{l}\text { LT @ Presyncope vs. } \\
\text { HT @ Submax }\end{array}$ \\
\hline \multicolumn{7}{|c|}{ ECG SIGNAL } \\
\hline $\mathrm{RRI}$ & & $643.6 \pm 22.0$ & $530.6 \pm 11.6$ & $587.1 \pm 113.4$ & $\mathrm{LT}>\mathrm{HT}$ & $\mathrm{LT}>\mathrm{HT}$ \\
\hline \multirow[t]{2}{*}{ HRV metrics } & & & & & Hypothesis & Hypothesis \\
\hline & & & & & $\mathrm{LT}=\mathrm{HT}$ & $\mathrm{LT}<\mathrm{HT}$ \\
\hline RRISD & $\mathrm{P}$ & $64.1 \pm 6.0$ & $38.9 \pm 2.3$ & $36.7 \pm 1.9$ & $\mathrm{LT}>\mathrm{HT}$ & $\mathrm{LT}>\mathrm{HT}$ \\
\hline RMSSD & $P$ & $23.9 \pm 5.8$ & $9.6 \pm 0.9$ & $14.8 \pm 1.2$ & $\mathrm{LT}>\mathrm{HT}$ & $\mathrm{LT}>\mathrm{HT}$ \\
\hline pNN50 & $P$ & $3.2 \pm 1.1$ & $0.9 \pm 0.2$ & $2.5 \pm 0.6$ & $\mathrm{LT}>\mathrm{HT}$ & $\mathrm{LT}=\mathrm{HT}$ \\
\hline SD1 & $P$ & $13.2 \pm 1.9$ & $6.8 \pm 0.6$ & $10.5 \pm 0.9$ & $\mathrm{LT}>\mathrm{HT}$ & $\mathrm{LT}=\mathrm{HT}$ \\
\hline SD2 & $P$ & $88.2 \pm 8.1$ & $54.0 \pm 3.2$ & $50.1 \pm 2.6$ & $\mathrm{LT}>\mathrm{HT}$ & $\mathrm{LT}>\mathrm{HT}$ \\
\hline \multicolumn{7}{|c|}{ FREQUENCY DOMAIN } \\
\hline $\mathrm{RRI} \mathrm{HF}$ & $\mathrm{P}$ & $216.1 \pm 88.7$ & $55.6 \pm 10.6$ & $188.9 \pm 38.1$ & $\mathrm{LT}>\mathrm{HT}$ & $\mathrm{LT}=\mathrm{HT}$ \\
\hline RRI LF & $P$ & $432.7 \pm 91.4$ & $302.4 \pm 62.3$ & $742.7 \pm 111.4$ & $\mathbf{L T}=\mathbf{H T}$ & $\mathrm{LT}=\mathrm{HT}$ \\
\hline \multicolumn{7}{|c|}{ COMPLEXITY DOMAIN } \\
\hline SampEn & $\mathrm{P}$ & $0.707 \pm 0.048$ & $0.57 \pm 0.029$ & $0.821 \pm 0.031$ & $\mathrm{LT}>\mathrm{HT}$ & $\mathrm{LT}=\mathrm{HT}$ \\
\hline LZEn & $P$ & $0.504 \pm 0.026$ & $0.420 \pm 0.017$ & $0.566 \pm 0.016$ & $\mathrm{LT}>\mathrm{HT}$ & $\mathbf{L T}<\mathbf{H T}$ \\
\hline FD-L* & $P$ & $1.572 \pm 0.026$ & $1.529 \pm 0.016$ & $1.701 \pm 0.011$ & $\mathbf{L T}=\mathbf{H T}$ & $\mathbf{L T}<\mathbf{H T}$ \\
\hline FD-DA & $P$ & $1.152 \pm 0.020$ & $1.128 \pm 0.012$ & $1.186 \pm 0.011$ & $\mathbf{L T}=\mathbf{H T}$ & $\mathrm{LT}=\mathrm{HT}$ \\
\hline SymDyn & $P$ & $0.521 \pm 0.018$ & $0.474 \pm 0.011$ & $0.580 \pm 0.010$ & $\mathrm{LT}>\mathrm{HT}$ & $\mathbf{L T}<\mathbf{H T}$ \\
\hline DisnEn & $P$ & $3.142 \pm 0.111$ & $2.846 \pm 0.067$ & $3.482 \pm 0.059$ & $\mathrm{LT}>\mathrm{HT}$ & $\mathbf{L T}<\mathbf{H T}$ \\
\hline DFA long* & I & $1.054 \pm 0.031$ & $1.100 \pm 0.025$ & $0.887 \pm 0.022$ & $\mathbf{L T}=\mathbf{H T}$ & $\mathbf{L T}<\mathbf{H T}$ \\
\hline
\end{tabular}

Data are shown in three groups: low tolerant (LT) at presyncope; high tolerant (HT) at presyncope; and HT at submax. Values (mean \pm SE) are shown for Heart Rate (HR), R-R interval (RRI), RRI standard deviation (RRISD), RRI root mean squared standard deviation (RMSSD), percentage adjacent RRIs varying by at least 50 ms (pNN50), Poincaré plot descriptors standard deviation 1 (SD1), and standard deviation 2 (SD2), SD1/SD2 ratio, SD2/SD1 ratio, complex demodulation high frequency (CDM HF), complex demodulation-low frequency (CDM LF), RRI high frequency power (HF), RRI low frequency power (LF), sample entropy (SampEn), Lempel-Ziv entropy (LZEn), fractal dimensions by curve length (FD-L), fractal dimensions by dispersion analysis (FD-DA), symbol dynamics entropy (SymDyn), normalized symbol dynamics entropy (DisnEn), long-range detrended fluctuation analysis (DFA long), short-range detrended fluctuation analysis (DFA short). P indicates HRV metrics values proportional to $R R I$ variability; I indicates HRV metrics values inversely proportional to RRI variability. Comparisons between groups are shown as $L T=H T$, $L T<H T$, and $L T>H T ;<,>$ denote significant difference between $L T$ and $H T$ group means $(p \leq 0.05)$; bold type comparisons support the related hypothesis. $*$ Denotes the metrics with responses to support both hypotheses.

variable in LT at presyncope (bold type in column 7 of Table 3 ). The responses of DFA long, FW, SD1/SD2, and FD-L were the only metrics to support both hypotheses, and are indicated by an asterisk $\left(^{*}\right)$ in Table 3. These four HRV metrics were further evaluated for their ability to distinguish individual LT and HT subjects.

\section{DFA LONG, FW, SD1/SD2, AND FD-L IN INDIVIDUAL LT AND HT SUBJECTS}

DFA long, FW, SD1/SD2, and FD-L in individual LT subjects at presyncope and individual HT subjects at submax are shown as box and whisker plots in Figures 3A-D. Statistical analysis of group averages (Table 3 ) reveal significant differences in the four
HRV measurements between LT and HT groups. However, when the measurements of DFA long, FW, SD1/SD2, and FD-L from individual subjects are graphically displayed (Figures 3A-D), it is evident that individual responses from LT subjects at presyncope are predominantly within the range of responses observed in HT subjects at submax despite the fact that LT subjects are on the verge of cardiovascular collapse and HT subjects are hemodynamically stable. The percentage of LT responses that were coincident with HT responses were $97 \%$ for DFA long and FW, $94 \%$ for SD1/SD2, and 85\% for FD-L. The sensitivities of DFA long, FW, SD1/SD2, and FD-L to identify individual LT subjects were low $(12,6,24,33 \%)$, and positive predictive values were $50,40,67$, and $73 \%$. 

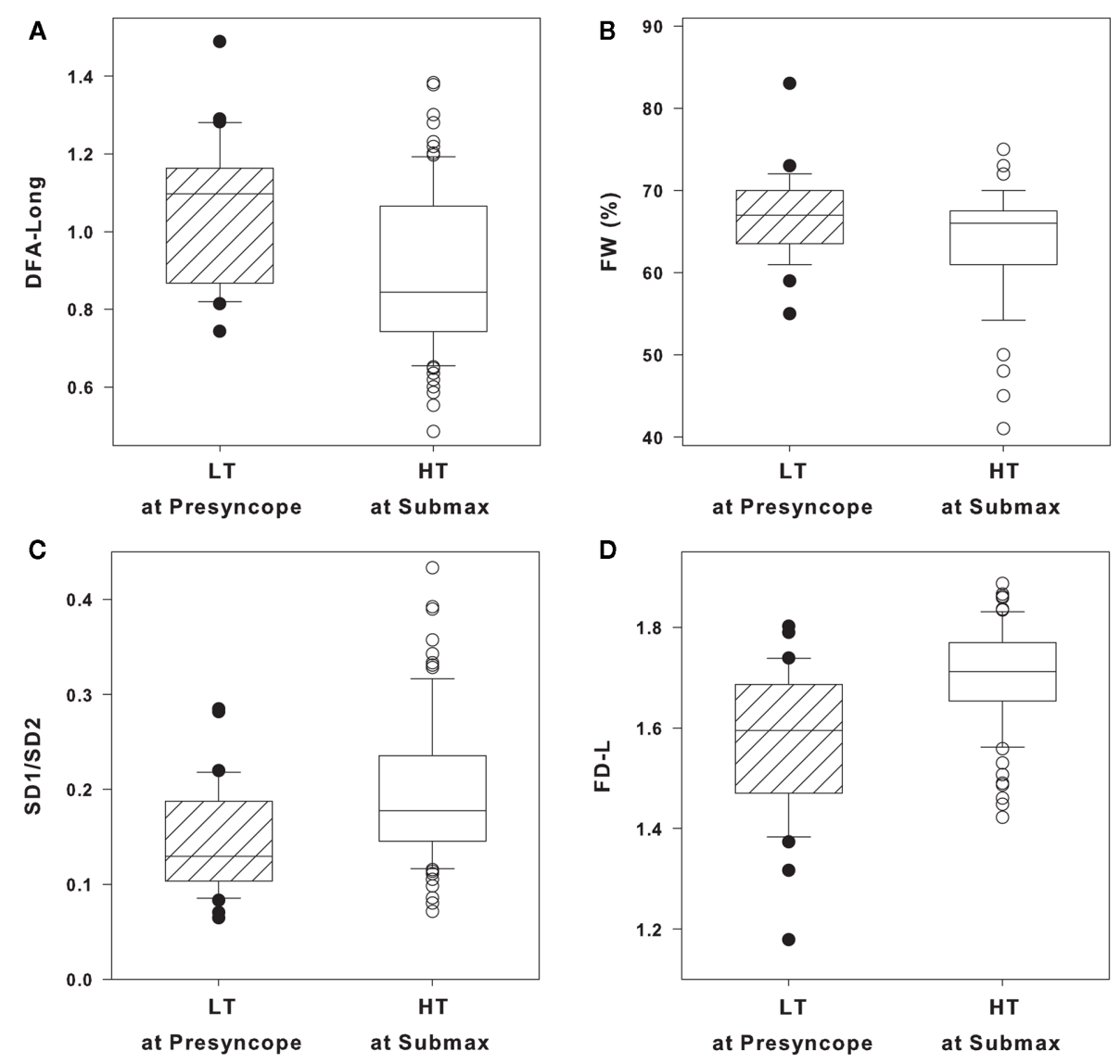

FIGURE 3 | (A) Long-range detrended fluctuation analysis (DFA long), (B) forbidden words (FW), (C) Poincare plot standard deviations ratio (SD1/SD2), and (D) fractal dimensions by curve length (FD-L) in low tolerant (LT) at presyncope (solid circles,

$n=33$ ) and high tolerant (HT) at submax (open circles, $n=87$ ) level of lower body negative pressure (LBNP). Data are shown as box (25th/75th percentiles) and whisker (90th/10th percentiles) plots with median value (black line).

\section{DISCUSSION}

To investigate the application of HRV monitoring for assessment of hemodynamic stability during hemorrhage, we used an experimental model to compare HRV metrics in LT and HT subjects during LBNP. In order for any specific HRV metric to be considered as a valid candidate for the assessment of cardiovascular stability in the context of central blood volume loss, two conditions must hold true using this model: (1) LT and HT subjects should display similar HRV at presyncope; and (2) HRV in LT subjects should be less (i.e., LT subjects would be less stable) than HRV in HT subjects at the point in time when LT subjects experienced presyncope. These conditions are based on the premise that hemodynamic instability reflects a specific physiological condition defined by the inability of cardiovascular mechanisms to adequately compensate for reduced central blood volume in all subjects independent of their tolerance, and that LT subjects are hemodynamically unstable at presyncope when HT subjects remain stable. Our results indicate that of the $20 \mathrm{HRV}$ metrics evaluated, only four metrics (DFA Long, FW, SD1/SD2, and FD-L; three of which were calculated by non-linear methods to assess RRI irregularity or complexity) supported both hypotheses.

We propose that a metric which can predict low tolerance in an individual subject prior to cardiovascular collapse would be optimal for use as a triage assist tool. Identification of LT patients is particularly important as the first responder will have less time to initiate effective treatment in this patient population compared with HT patients. Upon identifying the metrics which supported our initial hypotheses, we further evaluated DFA Long, FW, SD1/SD2, and FD-L on their utility as triage assist tools in individual subjects. First, we compared the individual responses from LT subjects at presyncope and HT subjects at submax to determine the overlap in responses between the two groups. Second, we assessed the accuracy of these HRV metrics in detecting LT subjects at presyncope from the total subject pool (sensitivity and positive predictive value).

While the group mean values of DFA Long, FW, SD1/SD2, and FD-L (Table 3) distinguished between LT at presyncope (unstable) and HT at submax (stable), the responses in individual LT and HT subjects varied extensively such that $85-97 \%$ of the LT responses overlapped with those of HT subjects (Figures 3A-D). In addition, the sensitivities of DFA Long, FW, SD1/SD2, and FD-L were 12, 6, 24 , and $33 \%$; while positive predictive values were $50,40,67$, and 
$73 \%$. Considering that identifying LT subjects by chance alone has $50 \%$ sensitivity and $50 \%$ positive predictive value, the accuracy of DFA Long and FW to identify LT subjects at presyncope was not much better than flipping a coin, or worse. Essentially, DFA Long and FW did not adequately identify a LT individual even when the subject was on the verge of cardiovascular collapse.

According to our results, FD-L had the highest positive predictive value (73\%), and thus had the highest potential for accurately assessing hypovolemia of all $20 \mathrm{HRV}$ metrics evaluated. However, the utility of FD-L to accurately monitor individual trauma patients may be limited by potentially high variability in individual responses. In the present study, under very controlled laboratory conditions, the sensitivity of FD-L to identify a LT subject was only $33 \%$, and the overlap of FD-L measurements from LT subjects and HT subjects was $85 \%$ (Figure 3D). Overall, these results indicate that the utility of FD-L as an accurate triage assisting tool for first responders is also limited.

Heart rate variability has been studied extensively during hemorrhage and trauma. HRV decreases with hypovolemia in human LBNP studies (Cooke and Convertino, 2005; Cooke et al., 2008) and in animal hemorrhage experiments (Batchinsky et al., 2007b, 2010). HRV has also been studied in pre-hospital environments during transport of trauma patients. By retrospective analysis of ECG data from actual trauma patients in transport to hospital care, a number of investigators (Cooke et al., 2006a,b; Batchinsky et al., 2007a; Cancio et al., 2008; Ong et al., 2008; King et al., 2009) have identified several HRV metrics that are associated with mortality or the need for a LSI. Importantly, however, all of these analyses were based on evaluation of group mean data only, with no consideration of the appropriateness of application to individual patients.

Despite the observation of depressed HRV in subsets of trauma patients, the use of HRV as a prognostic tool to assess the severity of hemorrhage or trauma is controversial for a number of important reasons. Ryan et al. (2010) evaluated the ability of numerous HRV metrics to track hypovolemia in individual subjects undergoing simulated hemorrhage with LBNP. They observed that when group means were evaluated several metrics correlated very well with reductions in stroke volume ( $r \geq 0.87$ ), but none of the HRV metrics consistently correlated with changes in stroke volume in individual subjects $(r \leq 0.49)$.

To further investigate the prognostic relevance of HRV, Rickards et al. (2010b) evaluated a wide range of HRV metrics and their association with the administration of LSIs in actual trauma patients who all had normal vital signs during transport. These patients would benefit most from identification of an early predictor of cardiovascular collapse, as their physiological status could not be accurately determined from currently available standard vital signs. Of the HRV metrics studied, only one metric, FD-L, was uniquely associated with the administration of a LSI. However, FD-L variance in both groups of patients was too high to accurately determine group membership (LSI vs. No-LSI) on an individual basis, and the number of false negatives identified with FD-L further limited the power of this metric as an accurate indicator of LSIs in individual trauma patients. Interestingly, FD-L also showed the highest positive predictive value for identifying LT subjects in the current study but, just as in trauma patients, the overlap between individual responses from LT and HT subjects was large (85\%), and we found the sensitivity (33\%) of this metric to be limited.

There are several technical factors associated with ECG monitoring which can limit the usefulness of HRV metrics in a prehospital setting. First, ECG signals must display normal sinus rhythm for accurate calculation of HRV, but trauma patients often develop arrhythmias such as premature atrial and ventricular contractions (Sethuraman et al., 2010). Second, ECG signals must have a low level of random noise, which usually results from motion artifact, an unavoidable consequence of patient transport in the pre-hospital setting. Third, the data from the ECG signal should be stationary, i.e., a relatively stable RRI signal without wide fluctuations from interventions or patient manipulation. Unfortunately, standard pre-hospital care typically requires extensive patient interventions and manipulation. Other factors which can also contribute to ECG non-stationarity such as disease, age, recreational drugs, medications, alcohol, smoking, and postural changes are widely present in the trauma patient population (Bilchick and Berger, 2006). Finally, the length of the ECG data set required for accurate measurements varies by HRV metric, and can range from 100 to 800 RRIs; which can be an inordinately long time in a trauma patient with rapidly changing physiological status (Acharya et al., 2006; Rickards et al., 2010a). Thus, the quality of HRV measurements are optimal when they can be calculated from extended, stable ECG recordings under standardized and very stable conditions; these conditions, however, are not typically encountered when first responders are treating trauma patients.

Historically, heart rate has been closely monitored in trauma patients because of the widely accepted notion that the magnitude of tachycardia reflects the degree of hypovolemia. However, the reliability of tachycardia in response to hypovolemia has been questioned as tachycardia can be absent in many trauma patients despite the development of hypotension associated with bleeding (Victorino et al., 2003; Brasel et al., 2007). In addition, the results of the current study indicate that tachycardia was a poor indicator of tolerance to hypovolemia because LT subjects at presyncope had lower heart rates than HT subjects at greater levels of central hypovolemia (Table 3). Based on the clinical doctrine that tachycardia signals a more severe state of hypovolemia and the approach of cardiovascular collapse, the HT subjects would have been erroneously identified as more hemodynamically unstable and at greater risk of developing circulatory shock than LT subjects. Although a change in cardiac rhythm may represent an adverse clinical status during hemorrhage, the results of the present study reinforce the unreliability of heart rate (and subsequently calculated metrics derived from heart rate such as HRV) for patient triage.

In summary, the results of the current study are consistent with the findings that ECG-derived metrics of HRV failed to provide reliable information about clinical status in individual subjects during progressive reductions in central blood volume similar to those experienced during hemorrhage (Ryan et al., 2010), or the need for LSIs in individual trauma patients (Rickards et al., 2010b). In the present study, heart rate and HRV metrics derived from ECG signals were found to be poor indicators of LT to hypovolemia even in the controlled experimental environment of simulated 
hemorrhage. This study raises further concern that monitoring heart rate or calculated derivatives of heart rate (e.g., HRV) will not reliably identify those patients who are least tolerant to hypovolemia and therefore at highest risk for early hemodynamic collapse during hemorrhage.

\section{ACKNOWLEDGMENTS}

We thank the research volunteers for their cheerful participation, Dr. James Aden for his assistance in statistical analysis, and

\section{REFERENCES}

Acharya, U. R., Joseph, K. P., Kannathal, N., Lim, C. M., and Suri, J. S. (2006). Heart rate variability: a review. Med. Biol. Eng. Comput. 44, 1031-1051.

Batchinsky, A. I., Cancio, L. C., Salinas, J., Kuusela, T., Cooke, W. H., Wang, J. J., Boehme, M., Convertino, V. A., and Holcomb, J. B. (2007a). Prehospital loss of R-to-R interval complexity is associated with mortality in trauma patients. J. Trauma 63, 512-518.

Batchinsky, A. I., Cooke, W. H., Kuusela, T., and Cancio, L. C. (2007b). Loss of complexity characterizes the heart rate response to experimental hemorrhagic shock in swine. Crit. Care Med. 35, 519-525.

Batchinsky, A. I., Skinner, J. E., Necsoiu, C., Jordan, B. S., Weiss, D., and Cancio, L. C. (2010). New measures of heart-rate complexity: effect of chest trauma and hemorrhage. J. Trauma 68, 1178-1185.

Bellamy, R. F. (1984). The causes of death in conventional land warfare: implications for combat casualty care research. Mil. Med. 149, 55-62.

Bilchick, K. C., and Berger, R. D. (2006). Heart rate variability. J. Cardiovasc. Electrophysiol. 17, 691-694.

Brasel, K., Guse, C., Gentilello, L. M., and Nirula, R. (2007). Heart Rate: is it truly a vital sign? J. Trauma 62 , 812-817.

Cancio, L. C., Batchinsky, A. I., Salinas, J., Kuusela, T. A., Convertino, V. A., Wade, C. E., and Holcomb, J. B. (2008). Heart-rate complexity for prediction of prehospital lifesaving interventions in trauma patients. $J$. Trauma 65, 813-819.

Champion, H., Bellamy, R., Roberts, P., and Leppaniemi, A. (2003). A profile of combat injury. J. Trauma 54, S13-S19.

Convertino, V. A. (2001). Lower body negative pressure as a tool for research in aerospace physiology and military medicine. J. Gravit. Physiol. 8, 1-14.

Convertino, V. A., Ryan, K. L., Rickards, C. A., Salinas, J., Mcmanus, J. G., Cooke, W. H., and Holcomb, J. B. (2008). Physiological and medical monitoring for en route care of combat casualties. J. Trauma 64, S342S353.

Convertino, V. A., and Sather, T. M. (2000). Effects of cholinergic and beta-adrenergic blockade on orthostatic tolerance in healthy subjects. Clin. Auton. Res. 10, 327-336.

Cooke, W. H., and Convertino, V. A. (2005). Heart rate variability and spontaneous baroreflex sequences: implications for autonomic monitoring during hemorrhage. J. Trauma 58, 798-805.

Cooke, W. H., Rickards, C. A., Ryan, K. L., and Convertino, V. A. (2008). Autonomic compensation to simulated hemorrhage monitored with heart period variability. Crit. Care Med. 36, 1892-1899.

Cooke, W. H., Ryan, K. L., and Convertino, V. A. (2004). Lower body negative pressure as a model to study progression to acute hemorrhagic 1249-1261.

Cooke, W. H., Salinas, J., Convertino, V. A., Ludwig, D. A., Hinds, D., Duke, J. H., Moore, F. A., and Holcomb, J. B. (2006a). Heart rate variability and its association with mortality in prehospital trauma patients. J. Trauma 60, 363-370.

Cooke, W. H., Salinas, J., Mcmanus, J. M., Ryan, K. L., Rickards, C. A., Holcomb, J. B., and Convertino, V. A. (2006b). Heart period variability in trauma patients may predict mortality and allow remote triage. Aviat. Space Environ. Med. 77, 1107-1112.

Grogan, E. L., Norris, P. R., Speroff, T., Ozdas, A., France, D. J., Harris, P. A., Jenkins, J. M., Stiles, R., Dittus, R. S., and Morris, J. A. (2005). Volatility: a new vital sign identified using a novel bedside monitoring strategy. J. Trauma 58, 7-14.

Heckbert, S. R., Vedder, N. B., Hoffman, W., Wilma, R. N., Winn, R. K., Hudson, L. D., Jurkovich, G. J., Copass, M. K., Harlan, J. M., Rice, C. L., and Maier, R. V. (1998). Outcome of hemorrhagic shock in trauma patients. J. Trauma 45, 545-549.

Jansen, J. R., Wesseling, K. T., Settels, J. J., and Schreuder, J. J. (1990). shock in humans. J. Appl. Physiol.96,

Mr. Gary Muniz for his excellent laboratory assistance. This study was funded by the United States Army Medical Research and Materiel Command.

\section{DISCLAIMER}

The opinions or assertions contained herein are the private views of the authors, and are not to be construed as official or as reflecting the views of the Department of the Army or the Department of Defense.

Continuous cardiac output monitoring by pulse contour during cardiac surgery. Eur. Heart J. 11(Supp. I), 26-32.

King, D. R., Ogilvie, M. P., Pereira, B. M., Chang, Y., Manning, R. J., Conner, J. A., Schulman, C. I., Mckenney, M. G., and Proctor, K. G. (2009). Heart rate variability as a triage tool in patients with trauma during prehospital helicopter transport. J. Trauma 67, 436-440.

Klemcke, H. G., Joe, B., Calderon, M. L., Rose, R., Oh, T., Aden, J., and Ryan, K. L. (2011). Genetic influences on survival time after severe hemorrhage in inbred rat strains. Physiol. Genomics 43, 758-765.

Malik, M. (1996). Task force of the European Society of Cardiology and the North American Society of Pacing and Electrophysiology. Heart rate variability: standards of measurement, physiological interpretation, and clinical use. Circulation 93, 1043-1065.

Morris, J. A. Jr., Norris, P. R., Ozdas, A., Waitman, L. R., Harrell, F. E. Jr., Williams, A. E., Cao, H., and Jenkins, J. M. (2006). Reduced heart rate variability: an indicator of cardiac uncoupling and diminished physiologic reserve in 1,425 trauma patients. J. Trauma 60, 1165-1173; discussion 1173-1164.

Norris, P. R., Anderson, S. M., Jenkins, J. M., Williams, A. E., and Morris, J. A. Jr. (2008). Heart rate multiscale entropy at three hours predicts hospital mortality in 3,154 trauma patients. Shock 23, 399-405.

Norris, P. R., Morris, J. A., Ozdas, A., Grogan, E. L., and Williams, A. E. (2005). Heart rate variability predicts trauma patient outcome as early as $12 \mathrm{~h}$ : implications for military and civilian triage. J. Surg. Res. 129, 122-128.

Ong, M. E., Padmanabhan, P., Chan, Y. H., Lin, Z., Overton, J., Ward, K. R., and Fei, D. Y. (2008). An observational, prospective study exploring the use of heart rate variability as a predictor of clinical outcomes in pre-hospital ambulance patients. Resuscitation 78, 289-297.
Parks, J. K., Elliott, A. C., Gentilello, L. M., and Shafi, S. (2006). Systemic hypotension is a late marker of shock after trauma: a validation study of Advanced Trauma Life Support principles in a large national sample. Am. J. Surg. 192, 727-731.

Pregibon, D. (1981). Logistic regression diagnostics. Ann. Stat. 9, 705-724.

Rickards, C. A., Ryan, K. L., and Convertino, V. A. (2010a). Characterization of common measures of heart period variability in healthy human subjects: implications for patient monitoring. J. Clin. Monit. Comput. 24, 61-70.

Rickards, C. A., Ryan, K. L., Ludwig, D. A., and Convertino, V. A. (2010b). Is heart period variability associated with the administration of lifesaving interventions in individual prehospital trauma patients with normal standard vital signs? Crit. Care Med. 38, 1666-1673.

Rickards, C. A., Ryan, K. L., Cooke, W. H., and Convertino, V. A. (2011). Tolerance to central hypovolemia: the influence of oscillations in arterial pressure and cerebral blood velocity. J. Appl. Physiol. 111, 1048-1058.

Ryan, K. L., Rickards, C. A., Ludwig, D. A., and Convertino, V. A. (2010). Tracking central hypovolemia with ecg in humans: cautions for the use of heart period variability in patient monitoring. Shock 33, 583-589.

Ryan, M. L., Thorson, C. M., Otero, C. A., Vu, T., and Proctor, K. G. (2011). Clinical applications of heart rate variability in the triage and assessment of traumatically injured patients. Anesthesiol. Res. Pract. 2011, Article ID: 416590.

Sather, T. M., Goldwater, D. J., Montgomery, L. D., and Convertino, V. A. (1986). Cardiovascular dynamics associated with tolerance to lower body negative pressure. Aviat. Space Environ. Med. 57, 413-419.

Sauaia, A., Moore, F. A., Moore, E. E., Moser, K. S., Brennan, R., Read, R. A., and Pons, P. T. (1995). Epidemiology of trauma deaths: a reassessment. $J$. Trauma 38, 185-193. 
Sethuraman, G., Ryan, K. L., Rickards, C. A., and Convertino, V. A. (2010). Ectopic beats in healthy humans and trauma patients: implications for use of heart period variability indices in medical monitoring. Aviat. Space Environ. Med. 81, 125-129.

Shoemaker, W. C., Montgomery, E. S., Kaplan, E., and Elwyn, D. H. (1973). Physiologic patterns in surviving and nonsurviving shock patients. Use of sequential cardiorespiratory variables in defining criteria for therapeutic goals and early warning of death. Arch. Surg. 106, 630-636.

Shoemaker, W. C., Wo, C. C., Lu, K., Chien, L. C., Bayard, D. S., Belzberg, H., Demetriades, D., and Jelliffe, R. W. (2006). Outcome prediction by a mathematical model based on noninvasive hemodynamic monitoring. J. Trauma 60, 82-90.

Thayer, J. F., and Sternberg, E. (2006). Beyond heart rate variability: vagal regulation of allostatic systems. Ann. N. Y. Acad. Sci. 1088, 361-372.

Victorino, G. P., Battistella, F. D., and Wisner, D. H. (2003). Does tachycardia correlate with hypotension after trauma? J. Am. Coll. Surg. 196, 679-684.

Ward, K. R., Tiba, M. H., Ryan, K. L., Filho, I. P., Rickards, C. A., Witten, T., Soller, B. R., Ludwig, D. A., and Convertino, V. A. (2010). Oxygen transport characterization of a human model of progressive hemorrhage. Resuscitation 81, 987-993.
Winchell, R. J., and Hoyt, D. B. (1996). Spectral analysis of heart rate variability in the ICU: a measure of autonomic function. J. Surg. Res. 63, 11-16.

Winchell, R. J., and Hoyt, D. B. (1997). Analysis of heart-rate variability: a noninvasive predictor of death and poor outcome in patients with severe head injury. J. Trauma 43 927-933.

Conflict of Interest Statement: The authors declare that the research was conducted in the absence of any commercial or financial relationships that could be construed as a potential conflict of interest.

Received: 25 July 2011; paper pending published: 22 August 2011; accepted: 01
November 2011; published online: 21 November 2011.

Citation: Hinojosa-Laborde C, Rickards CA, Ryan KL and Convertino VA (2011)

Heart rate variability during simulated hemorrhage with lower body negative pressure in high and low tolerant subjects. Front. Physio. 2:85. doi: 10.3389/fphys.2011.00085

This article was submitted to Frontiers in Clinical and Translational Physiology, a specialty of Frontiers in Physiology.

Copyright (c) 2011 Hinojosa-Laborde, Rickards, Ryan and Convertino. This is an open-access article subject to a nonexclusive license between the authors and Frontiers Media SA, which permits use, distribution and reproduction in other forums, provided the original authors and source are credited and other Frontiers conditions are complied with. 